\title{
LOCAL HARNACK ESTIMATE FOR MEAN CURVATURE FLOW IN EUCLIDEAN SPACE*
}

\author{
JIE WANG ${ }^{\dagger}$
}

Abstract. We obtain the local Harnack estimate of mean curvature flow in Euclidean space $\mathbb{R}^{n+1}$, under the condition $-m(t) g_{a b} \leq h_{a b} \leq M g_{a b}$, s.t. $0 \leq m(t) \leq M$, and $D_{t} m(t) \geq(n+3) m M^{2}$, on $t \in\left[0, \frac{\pi^{2}}{4(n+1) M^{2}}\right]$. As a corollary, we get a sharp gradient estimate of mean curvature in some directions.

Key words. Mean curvature flow, Local Harnack estimate.

AMS subject classifications. 53C21, 53C44

1. Introduction. The differential Harnack estimate of mean curvature flow was done by R.Hamilton in [1]. Recently, Hamilton find a new method to get local Harnack inequality for Ricci flow in [2].

TheOREM 1. (Hamilton's Local Harnack estimate for Ricci-flow) Let $M^{n}$ is a Riemannian manifold. $(M, g(t))$ is the solution of the Ricci-flow equation

$$
\frac{\partial}{\partial t} g_{i j}=-2 R_{i j}, \quad t \in[0, T)
$$

$U \subset M^{n}$ is an open set. On $U \times\left[0, t_{0}\right], t_{0}<T$ it satisfies the following curvature condition: $\exists C_{0}, \forall M>0$, where $M$ is a positive constant

$$
\begin{cases}-m(t)\left(g_{a c} g_{b d}-g_{a d} g_{b c}\right) \leq R_{a b c d} \leq M\left(g_{a c} g_{b d}-g_{a d} g_{b c}\right), & \\ 0 \leq m(t) \leq M & t \in\left[0, t_{0}\right] \\ m^{\prime}(t) \geq C_{0} m M & t \in\left[0, t_{0}\right]\end{cases}
$$

$O \in U$, set $C_{1}=M r^{2}$, s.t. $B_{r}\left(O, t_{0}\right) \subset \subset U$, then for $\forall(p, t) \in B_{\frac{r}{2}}\left(O, t_{0}\right) \times\left[t_{0}-\frac{r^{2}}{4}, t_{0}\right]$ and $\forall V \in T_{p} M^{n}$, we have

$$
D R(V)^{2} \leq C M^{2}\left(R c(V, V)+C m|V|^{2}\right) .
$$

Where $C$ only depends on $n, C_{1}$ is a positive constant.

Then by using the inequality, Hamilton get a theorem of curvature bound at finite distance for Ricci-flow in [3].

Motivated by his work, the author do a similar work in mean curvature flow. Maybe the work will be used in mean cuvature flow as the same way.

Let $M^{n}$ be a smooth manifold without boundary, and let $F_{0}: M^{n} \rightarrow \mathbb{R}^{n+1}$ a smooth immersion. Let

$$
F(\cdot, t): M^{n} \times[0, T) \rightarrow \mathbb{R}^{n+1}
$$

\footnotetext{
*Received February 13, 2008; accepted for publication July 23, 2008.

${ }^{\dagger}$ Centre of Mathematics and Science, Zhejiang University, Hangzhou, Zhejiang, 310027, P. R. China (ccdbb2004@yahoo.com.cn).
} 
be a one-parameter family of smooth hypersurface immersions in $\mathbb{R}^{n+1}$. We say that it is a solution to mean curvature flow if

$$
\frac{\partial}{\partial t} F(x, t)=H(x, t) \vec{\nu}, \quad x \in M^{n}, t>0
$$

where $H$ and $\vec{\nu}$ are mean curvature and unit inward unit normal respectively, so $H \vec{\nu}$ is the mean curvature vector.

Set $U \subset M^{n}$ is an open subset, and on $U \times\left[0, t_{0}\right]$, s.t. $t_{0}<T$, we have the curvature condition $-M g_{a b}(t) \leq H_{a b}(t) \leq M g_{a b}(t)$. Let $0 \leq R \leq \frac{\pi}{2 \sqrt{n+1} M}, O \in M^{n}$, $B_{R}(O, t)$ is a geodesic ball centered at $O, R$ is the radius at time $t$, s.t. $B_{R}(O, t)$ $\subset \subset U$ at $t \in\left[0, t_{0}\right]$. So we can set $M R=C_{0}$. Set $d_{t}(x)=d_{t}(x, O)$ is the geodesic distance function from $O$ to $x$ respect to $g_{i j}(t)$.

Through out the paper, we call the curvature condition

$$
(\star)\left\{\begin{array}{ll}
(1)-m(t) g_{a b}(t) \leq H_{a b}(t) \leq M g_{a b}(t) & \\
(2) 0 \leq m(t) \leq M & \text { on } t \in\left[0, R^{2}\right] \\
(3) D_{t} m(t) \geq(n+3) m M^{2} & \text { on } t \in\left[0, R^{2}\right]
\end{array} .\right.
$$

MaIn Theorem 1. If on $B_{R}(O, t) \times\left[0, R^{2}\right]$ the condition $(\star)$ is satisfied, then at $\forall(x, t) \in B_{\frac{R}{2}}(O, t) \times\left[0, R^{2}\right], \forall V \in T_{x} M^{n}$, we can find some constant $B>0$, depend only on $n$ and $C_{0}$, then the local Harnack estimate holds, $D_{t} H+m(t) H^{2}+2 D H(V)+$ $\left(H_{a b}+m(t) g_{a b}\right) V_{a} V_{b}+B M\left(1+\frac{R^{2}}{\left(R^{2}-4 d^{2}\right)^{2}}+\frac{1}{t}\right) \geq 0$.

Then we get a sharp gradient estimate of mean curvature:

COROLlary 1. Under the same condition of Main Theorem1, at point $\left(O, R^{2}\right)$,

we have

$$
\left\{\begin{array}{lr}
\text { (1) }|D H(V)|^{2} \leq C M^{3}\left(H_{a b}+m(t) g_{a b}\right) V_{a} V_{b}, & M \geq 1 \\
\text { (2) }|D H(V)|^{2} \leq C\left(H_{a b}+m(t) g_{a b}\right) V_{a} V_{b}, & 0 \leq M<1 \\
\text { (3) }|D H(V)|=0 & \text { if }\left[D_{t} H+m(t) H^{2}\right. \\
& \left.+B M\left(1+\frac{1}{R^{2}}+\frac{1}{t}\right)\right]\left(O, R^{2}\right)=0 \\
& \text { or }\left(H_{a b}+m(t) g_{a b}\right) V_{a} V_{b}\left(O, R^{2}\right)=0
\end{array},\right.
$$

$C$ is depend only on $n$ and $C_{0}$.

Now we introduce the structure of the paper. In section 2, we introduce the notations and conventions. In section 3, we use the Huisken-Ecker gradient estimate in [4] to get a proper form of gradient estimate of curvature under the curvature condition $(\star)$. In section 4 , we introduce a good extra term, it has positive lower bound and it play an important role in the local Harnack estimate. In section 5, we estimate the lower bound of another extra term in Harnack calculation, using the gradient estimate we got in section 3 . Then we use the good extra term to get local Harnack estimate under condition $(\star)$ in section 6 . In section 7 , we have some remarks to verify the inequality and the method are not trivial. 
2. Notations and conventions. $M$ is an $n$-dimensional manifold without boundary immersed in Euclidean space $\mathbb{R}^{n+1}$, it is parametrized locally by $X=\left\{x^{i}\right\}$ in $\mathbb{R}^{n}$, which $(i=1, \ldots, n)$. We denote $Y=\left\{y^{\alpha}\right\}$ in $\mathbb{R}^{n+1}(\alpha=1, \ldots, n+1), M$ is locally by $y^{\alpha}=F^{\alpha}\left(x^{i}\right)$. The tangent vectors on $M$ in $\mathbb{R}^{n+1}$ is denoted by $D_{i} Y=\frac{\partial Y}{\partial x^{i}}$. The Euclidean metric is $I=\left\{I_{\alpha \beta}\right\}$, then the induced metric $G=\left\{g_{i j}\right\}$ on M is

$$
g_{i j}=I\left(D_{i} Y, D_{j} Y\right)=I_{\alpha \beta} D_{i} y^{\alpha} D_{j} y^{\beta} .
$$

The unit normal $\vec{\nu}=\left\{N^{\alpha}\right\}$ is defined by

$$
I_{\alpha \beta} N^{\alpha} N^{\beta}=1 \quad \text { and } \quad I_{\alpha \beta} N^{\alpha} D_{i} y^{\beta}=0 .
$$

On the convex surfaces we take $\vec{\nu}$ to be inward. The metric $G=\left\{g_{i j}\right\}$ induces a LeviCivita connection $\Gamma=\left\{\Gamma_{j k}^{i}\right\}$ on $M$. So we can take covariant derivatives $D=\left\{D_{i}\right\}$ of tensors on $M$.

We denote $A=\left\{h_{i j}\right\}$ be the second fundamental form of $M, H=g^{i j} h_{i j}$ is the mean curvature.

3. Gradient Estimate of second fundamental form. In this section, we will use Hessian comparison theorem and Huisken-Ecker gradient estimate in [4] to get the gradient estimate in a proper form.

We know on $U \times\left[0, t_{0}\right]$, we have $-M g_{a b}(t) \leq h_{a b}(t) \leq M g_{a b}(t)$, then $R_{a b a b}=$ $H_{a a} H_{b b}-H_{a b} H_{a b}$. So we have

$$
-(n+1) M^{2} \leq R_{a b a b} \leq(n+1) M^{2} .
$$

We now use the Hessian comparison theorem in Chapter6 in [5].

TheOREm 2. (Hessian comparison theorem) Assume that $(M, g)$ satisfies $k \leq$ $\sec \leq K$. If $g_{r}$ represents the metric in the polar coordinates, then we have

$$
\frac{S n_{K}^{\prime}(r)}{S n_{K}(r)} g_{r} \leq H e s s(r) \leq \frac{S n_{k}^{\prime}(r)}{S n_{k}(r)} g_{r},
$$

where

$$
S n_{K}(r)=\left\{\begin{array}{ll}
\frac{1}{\sqrt{K}} \sin (\sqrt{K} r) & \text { if } K>0 \\
r & \text { if } K=0 \\
\frac{1}{\sqrt{|K|}} \sinh (\sqrt{|K|} r) & \text { if } K<0
\end{array} .\right.
$$

By this theorem, we get

$$
(n-1) \frac{S n_{K}^{\prime}(r)}{S n_{K}(r)} \leq \Delta r \leq(n-1) \frac{S n_{k}^{\prime}(r)}{S n_{k}(r)} .
$$

LEMma 1. If $U \subset M^{n}$ is an open subset, and on $U \times\left[0, t_{0}\right]$, s.t. $t_{0}<T$, we have the curvature condition $-M g_{a b}(t) \leq h_{a b}(t) \leq M g_{a b}(t)$. Let $0 \leq R \leq \frac{\pi}{2 \sqrt{n+1} M}$, $O \in M^{n}, B_{R}(O, t)$ is a geodesic ball centered at $O, R$ is the radius at time $t$. Set 
$d_{t}(x)=d_{t}(x, O)$ is the geodesic distance function from $O$ to $x$ respect to $g_{i j}(t)$. Then on $B_{R}(O, t) \times\left[0, R^{2}\right]$ we have

$$
|d \Delta d| \leq \frac{(n-1) \pi}{2} \cdot \frac{e^{\pi}+1}{e^{\pi}-1}
$$

Proof. Because $-(n+1) M^{2} \leq R_{a b a b} \leq(n+1) M^{2}$, then

$$
(n-1) d \frac{S n_{\sqrt{n+1} M}^{\prime}(d)}{S n_{\sqrt{n+1} M}(d)} \leq d \Delta d \leq(n-1) d \frac{S n_{-\sqrt{n+1} M}^{\prime}(d)}{S n_{-\sqrt{n+1} M}(d)},
$$

so we get

$$
(n-1)(\sqrt{n+1} M d) \operatorname{ctg}(\sqrt{n+1} M d) \leq d \Delta d \leq(n-1)(\sqrt{n+1} M d) \frac{e^{2 \sqrt{n+1} M d}+1}{e^{2 \sqrt{n+1} M d}-1} .
$$

It is easy to verify that $\lim _{x \rightarrow 0} x \operatorname{ctg} x=\lim _{x \rightarrow 0} x \operatorname{coth}(x)=1$, and on $[0, \pi), x \operatorname{ctg} x$ is a decreasing fuction, $x \operatorname{coth}(x)$ is a increasing funtion. When $d \leq R \leq \frac{\pi}{2 \sqrt{n+1} M}$, then $x=\sqrt{n+1} M d \leq \frac{\pi}{2}$, so $0 \leq d \Delta d(x, t) \leq(n-1) \frac{\pi}{2} \frac{e^{\pi}+1}{e^{\pi}-1},(x, t) \in B_{R}(O, t) \times\left[0, R^{2}\right]$. 口

Lemma 2. On $(x, t) \in B_{R}(O, t) \times\left[0, R^{2}\right]$,

$$
\left|\left(\frac{\partial}{\partial t}-\Delta\right) d^{2}\right| \leq C,\left|\nabla d^{2}\right|^{2}=4 d^{2}
$$

where $C$ is a positive constant depend only on $n$.

Proof. Because $\frac{\partial d^{2}}{\partial t}=2 d \frac{\partial d}{\partial t}=2 d \frac{d}{d t} \int_{\gamma} \sqrt{g_{i j} x^{i} x^{j}} d s=2 d \int_{\gamma}-H H_{i j} x^{i} x^{j} d s$. So $\left|\frac{\partial}{\partial t} d^{2}\right| \leq n M^{2} d^{2} \leq \frac{n \pi^{2}}{4(n+1)}$. In another hand, $\left|\Delta d^{2}\right|=\left.|2| D d\right|^{2}+2 d \Delta d|=| 2+(n-$ 1) $\frac{\pi}{2} \frac{e^{\pi}+1}{e^{\pi}-1} \mid \leq C$. So we have

$$
\left|\left(\frac{\partial}{\partial t}-\Delta\right) d^{2}\right| \leq C
$$

And $\left|\nabla d^{2}\right|^{2}=\left.\left|4 d^{2}\right| \nabla d\right|^{2} \mid=4 d^{2}$.

Now we state the Theorem3.7 in [4] as follow.

Theorem 3. (Theorem 3.7 in [4]) Let $r=r(x, t) \geq 0$, satisfies

$$
\left|\left(\frac{\partial}{\partial t}-\Delta\right) r\right| \leq C(n),|\nabla r|^{2} \leq C(n) r .
$$

Let $R>0$, s.t. $\left\{x \in M_{t} \mid r(x, t) \leq R^{2}\right\}$ is compact for $t \in[0, T]$. Then for $0 \leq \theta<1$, $t \in[0, T]$ and integers $l \geq 0$, we have the estimate

$$
\sup _{\left\{x \in M_{t} \mid r(x, t) \leq \theta R^{2}\right\}}\left|\nabla^{l} A\right|^{2} \leq C(n, \theta) \sup _{\left\{x \in M_{t} \mid r(x, t) \leq \theta R^{2}, s \in[0, t]\right\}}|A|^{2}\left(1+\frac{1}{R^{2}}+\frac{1}{t}\right)^{l} .
$$

We see if $r(x, t)=d_{t}^{2}(x, O), \theta=\frac{1}{4}$, then 


$$
\sup _{\left\{x \in M_{t} \mid d_{t}(O, x) \leq \frac{R}{2}\right\}}\left|\nabla^{l} A\right|^{2} \leq C(n) M^{2}\left(1+\frac{1}{R^{2}}+\frac{1}{t}\right)^{l} .
$$

So we get the gradient estimate in a proper form as follow.

Corollary 2 (Derivative estimates of second fundamental form). If on $B_{R}(O, t) \times\left[0, R^{2}\right]$, where $0 \leq R \leq \frac{\pi}{2 \sqrt{n+1} M}$, holds the curvature condition $(\star)$, then we have on $B_{\frac{R}{2}}(O, t) \times\left[0, R^{2}\right]$

$$
\begin{gathered}
|\nabla A|^{2} \leq C M^{2}\left(1+\frac{1}{R^{2}}+\frac{1}{t}\right), \\
\left|\nabla^{2} A\right|^{2} \leq C M^{2}\left(1+\frac{1}{R^{2}}+\frac{1}{t}\right)^{2} .
\end{gathered}
$$

Where $C$ is a positive constant depend only on $n$.

4. Good extra term. In this section, we will change the Harnack quantities in [1], then we will find a good positive extra term.

We now recall the Harnack quantities in [1].

$$
\begin{gathered}
X_{a}=D_{a} H+H_{a b} V_{b}, \\
Y_{a b}=D_{a} V_{b}-H H_{a b}, \\
Z=D_{t} H+2 V_{a} D_{a} H+H_{a b} V_{a} V_{b}, \\
W_{a b}=D_{t} H_{a b}+V_{c} D_{c} H_{a b}, \\
W=D_{t} H+V_{c} D_{c} H, \\
U_{a}=\left(D_{t}-\Delta\right) V_{a}+H_{a b} D_{b} H . \\
\left(D_{t}-\Delta\right) Z=\left|H_{a b}\right|^{2} Z+2 X_{a} U_{a}-2 H_{b c} Y_{a b} Y_{a c}-4 W_{a b} Y_{a b} .
\end{gathered}
$$

We now change the $Y_{a b}$ to $\widetilde{Y_{a b}}=D_{a} V_{b}-H H_{a b}+\widetilde{E_{a b}}$.

Then

$$
\begin{aligned}
& \left(D_{t}-\Delta\right) Z \\
= & \left|H_{a b}\right|^{2} Z+2 X_{a} U_{a}-2 H_{b c} \widetilde{Y_{a b}} \widetilde{Y_{a c}}-4 W_{a b} \widetilde{Y_{a b}}+4 H_{b c} \widetilde{E_{a c}} \widetilde{Y_{a b}}+4 \widetilde{E_{a b}} W_{a b}-2 H_{b c} \widetilde{E_{a b}} \widetilde{E_{a c}} .
\end{aligned}
$$

We set $\widetilde{E_{a b}}=H_{b c}^{-1} W_{a c}$. So

$$
\begin{aligned}
& -4 W_{a b} \widetilde{Y_{a b}}+4 H_{b c} \widetilde{E_{a c}} \widetilde{Y_{a b}} \\
= & -4 W_{a b} \widetilde{Y_{a b}}+4 H_{b c} H_{c e}^{-1} W_{a e} \widetilde{Y_{a b}} \\
= & -4 W_{a b} \widetilde{Y_{a b}}+4 W_{a b} \widetilde{Y_{a b}} \\
= & 0,
\end{aligned}
$$


and

$$
\begin{aligned}
& 4 \widetilde{E_{a b}} W_{a b}-2 H_{b c} \widetilde{E_{a b}} \widetilde{E_{a c}} \\
= & 4 H_{b c}^{-1} W_{a c} W_{a b}-2 H_{b c} H_{b e}^{-1} W_{a e} H_{c f}^{-1} W_{a f} \\
= & 4 H_{b c}^{-1} W_{a c} W_{a b}-2 H_{b c}^{-1} W_{a c} W_{a b} \\
= & 2 H_{b c}^{-1} W_{a c} W_{a b} .
\end{aligned}
$$

So

$$
\begin{aligned}
\left(D_{t}-\Delta\right)(Z+\varphi)= & \left|H_{a b}\right|^{2}(Z+\varphi)+2 X_{a} U_{a}-2 H_{b c} \widetilde{Y_{a b}} \widetilde{Y_{a c}} \\
& +\left[\left(D_{t}-\Delta\right) \varphi+2 H_{b c}^{-1} W_{a c} W_{a b}-\left|H_{a b}\right|^{2} \varphi\right] .
\end{aligned}
$$

When $0 \leq H_{a b} \leq M g_{a b}$, we see $2 H_{b c}^{-1} W_{a c} W_{a b} \geq \frac{2}{M} W_{a b} W_{a b}$. So it is a good positive term to make $\left[\left(D_{t}-\Delta\right) \varphi+2 H_{b c}^{-1} W_{a c} W_{a b}-\left|H_{a b}\right|^{2} \varphi\right]$ positive. We will show the details in section 6 .

5. Estimate of another extra term. Under the condition $(\star)$, we will have another extra term, we call it $C N S$. So in this section we will estimate it.

First, under the condition $(\star)$, we change the quantities in last section again as follow.

$$
\begin{gathered}
\widetilde{H_{a b}}=H_{a b}+m(t) g_{a b}, \\
\widetilde{X_{a}}=D_{a} H+\widetilde{H_{a b}} V_{b}, \\
\widetilde{Y_{a b}}=D_{a} V_{b}-H H_{a b}+\widetilde{E_{a b}}, \\
\widetilde{Z}=D_{t} H+m(t) H^{2}+2 V_{a} D_{a} H+\widetilde{H_{a b}} V_{a} V_{b}, \\
\widetilde{W_{a b}}=D_{t} H_{a b}+m H H_{a b}+V_{c} D_{c} H_{a b}, \\
\widetilde{W}=D_{t} H+m H^{2}+V_{c} D_{c} H, \\
\widetilde{U_{a}}=\left(D_{t}-\Delta\right) V_{a}+\widetilde{H_{a b}} D_{b} H .
\end{gathered}
$$

We will define $\widetilde{E_{a b}}$ later.

Recall the equation

$$
\begin{aligned}
\left(D_{t}-\Delta\right) Z= & \left|H_{a b}\right|^{2} Z+2 X_{a} U_{a}-2 H_{b c} \widetilde{Y_{a b}} \widetilde{Y_{a c}}-4 W_{a b} \widetilde{Y_{a b}} \\
& +4 H_{b c} \widetilde{E_{a c}} \widetilde{Y_{a b}}+4 \widetilde{E_{a b}} W_{a b}-2 H_{b c} \widetilde{E_{a b}} \widetilde{E_{a c}} .
\end{aligned}
$$

We hope $\left(D_{t}-\Delta\right) \widetilde{Z}$ have following form,

$$
\begin{aligned}
\left(D_{t}-\Delta\right) \widetilde{Z}= & \left|H_{a b}\right|^{2} \widetilde{Z}+2 \widetilde{X_{a}} \widetilde{U_{a}}-2 \widetilde{H_{b c}} \widetilde{Y_{a b}} \widetilde{Y_{a c}}-4 \widetilde{W_{a b}} \widetilde{Y_{a b}} \\
& +4 \widetilde{H_{b c}} \widetilde{E_{a c}} \widetilde{Y_{a b}}+4 \widetilde{E_{a b}} \widetilde{W_{a b}}-2 \widetilde{H_{b c}} \widetilde{E_{a b}} \widetilde{E_{a c}}+C N S .
\end{aligned}
$$


So we get

$$
\begin{aligned}
C N S= & \left(D_{t}-\Delta\right)(\widetilde{Z}-Z)+\left|H_{a b}\right|^{2}(Z-\widetilde{Z})+2\left(X_{a} U_{a}-\widetilde{X_{a}} \widetilde{U_{a}}\right)+2\left(\widetilde{H_{b c}}-H_{b c}\right) \widetilde{Y_{a b}} \widetilde{Y_{a c}} \\
& +4 \widetilde{Y_{a b}}\left(\widetilde{W_{a b}}-W_{a b}\right)+4\left(H_{b c}-\widetilde{H_{b c}}\right) \widetilde{E_{a c}} \widetilde{Y_{a b}}+4 \widetilde{E_{a b}}\left(W_{a b}-\widetilde{W_{a b}}\right) \\
& +2\left(\widetilde{H_{b c}}-H_{b c}\right) \widetilde{E_{a b}} \widetilde{E_{a c}} .
\end{aligned}
$$

Now we calculate them carefully as follow.

1.

$$
\begin{aligned}
\left(D_{t}-\Delta\right)(\widetilde{Z}-Z)= & \left(D_{t}-\Delta\right)\left(m H^{2}+m|V|^{2}\right) \\
= & \left(D_{t} m\right) H^{2}+\left(D_{t} m\right)|V|^{2}-2 m|D H|^{2}-2 m|D V|^{2} \\
& +2 m H\left(D_{t}-\Delta\right) H+2 m V_{a}\left(D_{t}-\Delta\right) V_{a} .
\end{aligned}
$$

2.

$$
\left|H_{a b}\right|^{2}(Z-\widetilde{Z})=-m\left|H_{a b}\right|^{2} H^{2}-m\left|H_{a b}\right|^{2}|V|^{2} .
$$

3.

$$
\begin{aligned}
& 2\left(X_{a} U_{a}-\widetilde{X_{a}} \widetilde{U_{a}}\right) \\
= & -2 m|D H|^{2}-4 m H_{a b} V_{b} D_{a} H-2 m V_{a}\left(D_{t}-\Delta\right) V_{a}-2 m^{2} V_{a} D_{a} H .
\end{aligned}
$$

4.

$$
2\left(\widetilde{H_{b c}}-H_{b c}\right) \widetilde{Y_{a b}} \widetilde{Y_{a c}}=2 m \widetilde{Y_{a b}} \widetilde{Y_{a b}}
$$

5.

$$
4 \widetilde{Y_{a b}}\left(\widetilde{W_{a b}}-W_{a b}\right)=4 m H H_{a b} \widetilde{Y_{a b}}
$$

6.

$$
4\left(H_{b c}-\widetilde{H_{b c}}\right) \widetilde{E_{a c}} \widetilde{Y_{a b}}=-4 m \widetilde{E_{a b}} \widetilde{Y_{a b}}
$$

7.

$$
\widetilde{4 E_{a b}}\left(W_{a b}-\widetilde{W_{a b}}\right)=-4 m H H_{a b} \widetilde{E_{a b}}
$$

8.

$$
2\left(\widetilde{H_{b c}}-H_{b c}\right) \widetilde{E_{a b}} \widetilde{E_{a c}}=2 m \widetilde{E_{a b}} \widetilde{E_{a b}} .
$$

So we get

$$
\begin{aligned}
C N S= & \left(D_{t} m\right) H^{2}+\left(D_{t} m\right)|V|^{2}-2 m|D H|^{2}-2 m|D V|^{2}+2 m H\left(D_{t}-\Delta\right) H \\
& +2 m V_{a}\left(D_{t}-\Delta\right) V_{a}-m\left|H_{a b}\right|^{2} H^{2}-m\left|H_{a b}\right|^{2}|V|^{2}-2 m|D H|^{2} \\
& -4 m H_{a b} V_{b} D_{a} H-2 m V_{a}\left(D_{t}-\Delta\right) V_{a}-2 m^{2} V_{a} D_{a} H+2 m \widetilde{Y_{a b}} \widetilde{Y_{a b}} \\
& +4 m H H_{a b} \widetilde{Y_{a b}}-4 m \widetilde{E_{a b}} \widetilde{Y_{a b}}-4 m H H_{a b} \widetilde{E_{a b}}+2 m \widetilde{E_{a b}} \widetilde{E_{a b}} .
\end{aligned}
$$

Because

$$
\begin{aligned}
2 m \widetilde{Y_{a b}} \widetilde{Y_{a b}}-4 m \widetilde{E_{a b}} \widetilde{Y_{a b}}+2 m \widetilde{E_{a b}} \widetilde{E_{a b}} & =2 m\left(\widetilde{Y_{a b}}-\widetilde{E_{a b}}\right)^{2} \\
& =2 m\left(D_{a} V_{b}-H H_{a b}\right)^{2} \\
& =2 m|D V|^{2}-4 m D_{a} V_{a} H H_{a b}+2 m H^{2}\left|H_{a b}\right|^{2} .
\end{aligned}
$$


and

$$
4 m H H_{a b} \widetilde{Y_{a b}}-4 m H H_{a b} \widetilde{E_{a b}}=4 m D_{a} V_{b} H H_{a b}-4 m H^{2}\left|H_{a b}\right|^{2} .
$$

So

$$
\begin{aligned}
& 2 m \widetilde{Y_{a b}} \widetilde{Y_{a b}}+4 m H H_{a b} \widetilde{Y_{a b}}-4 m \widetilde{E_{a b}} \widetilde{Y_{a b}}-4 m H H_{a b} \widetilde{E_{a b}}+2 m \widetilde{E_{a b}} \widetilde{E_{a b}} \\
= & 2 m|D V|^{2}-2 m H^{2}\left|H_{a b}\right|^{2} .
\end{aligned}
$$

On the other hand

$$
\begin{aligned}
-4 m H_{a b} V_{b} D_{a} H & \geq-2 m H_{a c} H_{a b} V_{c} V_{b}-2 m|D H|^{2} \\
& \geq-2 m M^{2}|V|^{2}-2 m|D H|^{2},
\end{aligned}
$$

and

$$
-2 m^{2} V_{a} D_{a} H \geq-m^{3}|V|^{2}-m|D H|^{2} .
$$

So we get on $B_{\frac{R}{2}}(O, t) \times\left[0, R^{2}\right]$

$$
\begin{aligned}
C N S & =\left(D_{t} m-m\left|H_{a b}\right|^{2}\right) H^{2}+|V|^{2}\left(D_{t} m-m\left|H_{a b}\right|^{2}-2 m M^{2}-m^{3}\right)-7 m|D H|^{2} \\
& \geq\left(D_{t} m-n m M^{2}\right) H^{2}+|V|^{2}\left(D_{t} m-(n+3) m M^{2}\right)-7 m|D H|^{2} \\
& \geq-C m M^{2}\left(1+\frac{1}{R^{2}}+\frac{1}{t}\right) .
\end{aligned}
$$

The last inequality is hold by curvature condition $(\star)$.

6. Local Harnack estimate for curvature condition $(\star)$. In this section we will prove the local Harnack estimate for curvature condition $(\star)$.

If we add some fucntion $\varphi$, and set $\widetilde{E_{a b}}={\widetilde{H_{b c}}}^{-1} W_{a c}$, then we get the equality

$$
\begin{aligned}
\left(D_{t}-\Delta\right)(\widetilde{Z}+\varphi)= & \left|H_{a b}\right|^{2}(\widetilde{Z}+\varphi)+2 \widetilde{X_{a}} \widetilde{U_{a}}-2 \widetilde{H_{b c}} \widetilde{Y_{a b}} \widetilde{Y_{a c}}+2 \widetilde{H_{a b}}-1 \widetilde{W_{a c}} \widetilde{W_{b c}} \\
& +C N S+\left(D_{t}-\Delta\right) \varphi-\left|H_{a b}\right|^{2} \varphi
\end{aligned}
$$

If $-m(t) g_{a b}(t) \leq H_{a b}(t) \leq M g_{a b}(t)$, then $0 \leq \widetilde{H_{a b}} \leq(M+m(t)) g_{a b}$, so ${\widetilde{H_{a b}}}^{-1} \geq$ $\frac{1}{M+m(t)} g_{a b}$.

So

$$
\begin{aligned}
2 \widetilde{H_{a b}}-1 \widetilde{W_{a c}} \widetilde{W_{b c}} \geq & \frac{2}{M+m(t)} \widetilde{W_{a b}} \widetilde{W_{a b}} \\
= & \frac{2}{M+m(t)}\left(\widetilde{W_{a b}}+\frac{\varphi}{n} g_{a b}\right)^{2}-\frac{4}{M+m(t)}(\widetilde{W}+\varphi) \frac{\varphi}{n} \\
& +\frac{2}{M+m(t)} \frac{\varphi^{2}}{n} .
\end{aligned}
$$

Then

$$
\begin{aligned}
& \left(D_{t}-\Delta\right)(\widetilde{Z}+\varphi) \\
\geq & \left|H_{a b}\right|^{2}(\widetilde{Z}+\varphi)+2 \widetilde{X_{a}} \widetilde{U_{a}}-2 \widetilde{H_{b c}} \widetilde{Y_{a b}} \widetilde{Y_{a c}}+\frac{2}{M+m(t)}\left|\widetilde{W_{a b}}+\frac{\varphi}{n} g_{a b}\right|^{2} \\
& -\frac{4}{M+m(t)}(\widetilde{W}+\varphi) \frac{\varphi}{n}+\left[\left(D_{t}-\Delta\right) \varphi+\frac{2}{M+m(t)} \frac{\varphi^{2}}{n}-\left|H_{a b}\right|^{2} \varphi+C N S\right] .
\end{aligned}
$$


Now we prove Main theorem 1.

Proof of the Main Theorem 1. Let $\varphi=B M\left(1+\frac{R^{2}}{\left(R^{2}-4 d^{2}\right)^{2}}+\frac{1}{t}\right), B$ will be chosen later. We see $\varphi=+\infty$ at $B_{\frac{R}{2}}(O, 0) \cup \partial B_{\frac{R}{2}}(O, t), t \in\left[0, R^{2}\right]$. If $\widetilde{Z}+\varphi$ attain zero in $B_{\frac{R}{2}}(O, t) \times\left[0, R^{2}\right]$ at $\left(x_{0}, t_{0}, V\right)$ for the first time, then it must be $\left(x_{0}, t_{0}\right) \in \operatorname{int}\left(B_{\frac{R}{2}}(O, t)\right) \times\left(0, R^{2}\right]$. And we know

$$
0=\left.\frac{\partial(\widetilde{Z}+\varphi)(V+s \widetilde{V})}{\partial s}\right|_{s=0}=2 \sum_{a} \widetilde{X_{a}} \widetilde{V_{a}} \text {, for } \forall \widetilde{V} \in T_{x_{0}} M^{n} \text {. }
$$

So $\widetilde{X_{a}}=0$. On the other hand, we see $\widetilde{Z}+\varphi-\widetilde{X_{a}} V_{a}=\widetilde{W}+\varphi=0$. We set the expansion of $V$ as $\widetilde{Y_{a b}}=0$, and we see $C N S \geq-C m M^{2}\left(1+\frac{1}{R^{2}}+\frac{1}{t}\right)$ on $B_{\frac{R}{2}}(O, t) \times\left[0, R^{2}\right]$. Now we get

$$
\left(D_{t}-\Delta\right)(\widetilde{Z}+\varphi) \geq\left(D_{t}-\Delta\right) \varphi+\frac{2}{M+m(t)} \frac{\varphi^{2}}{n}-\left|H_{a b}\right|^{2} \varphi-C m M^{2}\left(1+\frac{1}{R^{2}}+\frac{1}{t}\right) .
$$

If we can hold the right hand side $>0$, then the theorem holds. We calculate the R.H.S. as follow.

1. $D_{t} \varphi=D_{t}\left[B M\left(1+\frac{R^{2}}{\left(R^{2}-4 d^{2}\right)^{2}}+\frac{1}{t}\right)\right]=B M\left(\frac{16 R^{2} d D_{t} d}{\left(R^{2}-4 d^{2}\right)^{3}}-\frac{1}{t^{2}}\right)$. Because $\left|D_{t} d\right|=$ $\left|D_{t} \int_{\gamma} \sqrt{g_{i j} x^{i} x^{j}} d s\right|=\left|\int_{\gamma}-H H_{i j} x^{i} x^{j} d s\right| \leq C M^{2} d$, so

$$
\begin{aligned}
D_{t} \varphi & \geq B M\left(-\frac{16 R^{2} d^{2} C M^{2}}{\left(R^{2}-4 d^{2}\right)^{3}}-\frac{1}{t^{2}}\right) \\
& \geq B M\left(-\frac{16 R^{2} d^{2}\left(R^{2}-4 d^{2}\right) C M^{2}}{\left(R^{2}-4 d^{2}\right)^{4}}-\frac{1}{t^{2}}\right) \\
& \geq B M\left(-\frac{C R^{4}}{\left(R^{2}-4 d^{2}\right)^{4}}-\frac{1}{t^{2}}\right) \\
& \geq-\frac{C}{B M} \varphi^{2} .
\end{aligned}
$$

2.

$$
\begin{aligned}
-\Delta \varphi & =-B M\left(\frac{16 R^{2}|D d|^{2}+16 R^{2} d \Delta d}{\left(R^{2}-4 d^{2}\right)^{3}}+\frac{384 R^{2} d^{2}|D d|^{2}}{\left(R^{2}-4 d^{2}\right)^{4}}\right) \\
& \geq-B M\left(\frac{C R^{2}}{\left(R^{2}-4 d^{2}\right)^{3}}+\frac{C R^{4}}{\left(R^{2}-4 d^{2}\right)^{4}}\right) \\
& \geq-B M\left(\frac{C R^{4}}{\left(R^{2}-4 d^{2}\right)^{4}}\right) \\
& \geq-\frac{C}{B M} \varphi^{2} .
\end{aligned}
$$

3. $\frac{2}{M+m(t)} \frac{\varphi^{2}}{n} \geq \frac{1}{M} \frac{\varphi^{2}}{n}$.

4. $-C M^{2} \varphi \geq-\frac{C}{B M} \varphi^{2}$.

5. $-C m M^{2}\left(1+\frac{1}{R^{2}}+\frac{1}{t}\right) \geq-\frac{C}{B^{2} M} \varphi^{2}$. 
So we get

$$
\begin{aligned}
\left(D_{t}-\Delta\right)(\widetilde{Z}+\varphi) & \geq-\frac{C}{B M} \varphi^{2}+\frac{1}{M} \frac{\varphi^{2}}{n}-\frac{C}{B^{2} M} \varphi^{2} \\
& \geq \frac{\varphi^{2}}{n M B^{2}}\left(B^{2}-n B C-n C\right) \\
& >0,
\end{aligned}
$$

when $B>\frac{n C+\sqrt{n^{2} C^{2}+4 n C}}{2}$.

Now we can prove the corollary 1.

Proof of Corollary 1. At $\left(O, R^{2}\right)$, for $\forall V \in T_{O} M^{n}$, we have

$$
D_{t} H+m(t) H^{2}+2 D H(V)+\left(H_{a b}+m(t) g_{a b}\right) V_{a} V_{b}+B M\left(1+\frac{1}{R^{2}}+\frac{1}{t}\right) \geq 0 .
$$

Then $|2 D H(V)| \leq D_{t} H+m(t) H^{2}+\widetilde{H_{a b}} V_{a} V_{b}+B M\left(1+\frac{1}{R^{2}}+\frac{1}{t}\right)$. And if $V=0$, we see $D_{t} H+m(t) H^{2}+B M\left(1+\frac{1}{R^{2}}+\frac{1}{t}\right) \geq 0$.

Case 1. If $D_{t} H+m(t) H^{2}+B M\left(1+\frac{1}{R^{2}}+\frac{1}{t}\right)>0, \widetilde{H_{a b}} V_{a} V_{b}>0$, then we can find $\lambda \in \mathbb{R}^{+}$, s.t. $\left[D_{t} H+m(t) H^{2}+B M\left(1+\frac{1}{R^{2}}+\frac{1}{t}\right)\right]\left(O, R^{2}\right)=\lambda^{2} \widetilde{H_{a b}} V_{a} V_{b}\left(O, R^{2}\right)$.

So

$$
\begin{aligned}
|2 D H(\lambda V)|^{2} & \leq\left[D_{t} H+m(t) H^{2}+2 D H(V)+\lambda^{2} \widetilde{H_{a b}} V_{a} V_{b}+B M\left(1+\frac{1}{R^{2}}+\frac{1}{t}\right)\right]^{2} \\
& \leq 4\left(D_{t} H+m(t) H^{2}+B M\left(1+\frac{1}{R^{2}}+\frac{1}{t}\right)\right) \lambda^{2} \widetilde{H_{a b}} V_{a} V_{b}
\end{aligned}
$$

so

$$
|D H(V)|^{2} \leq\left(D_{t} H+m(t) H^{2}+B M\left(1+\frac{1}{R^{2}}+\frac{1}{t}\right)\right) \widetilde{H_{a b}} V_{a} V_{b}
$$

Case 2. If $D_{t} H+m(t) H^{2}+B M\left(1+\frac{1}{R^{2}}+\frac{1}{t}\right)=0, \widetilde{H_{a b}} V_{a} V_{b}>0$, then for $\forall \varepsilon>0$, we can find $\lambda \in \mathbb{R}^{+}$, s.t. $\varepsilon=\lambda^{2} \widetilde{H_{a b}} V_{a} V_{b}\left(O, R^{2}\right)$. So

$$
\begin{aligned}
|2 D H(\lambda V)|^{2} & <\left[\varepsilon+\lambda^{2} \widetilde{H_{a b}} V_{a} V_{b}\right]^{2} \\
& =4 \varepsilon \lambda^{2} \widetilde{H_{a b}} V_{a} V_{b},
\end{aligned}
$$

let $\varepsilon \rightarrow 0$, we have $|D H(V)|=0$.

Case 3. If $D_{t} H+m(t) H^{2}+B M\left(1+\frac{1}{R^{2}}+\frac{1}{t}\right)>0, \widetilde{H_{a b}} V_{a} V_{b}=0$, then for $\forall \varepsilon>0$, we an find $\lambda \in \mathbb{R}^{+}$, s.t. $D_{t} H+m(t) H^{2}+B M\left(1+\frac{1}{R^{2}}+\frac{1}{t}\right)=\varepsilon \lambda^{2} g_{a b} V_{a} V_{b}\left(O, R^{2}\right)$.

So

$$
|2 D H(\lambda V)|^{2}<4\left[D_{t} H+m(t) H^{2}+B M\left(1+\frac{1}{R^{2}}+\frac{1}{t}\right)\right] \varepsilon \lambda^{2} g_{a b} V_{a} V_{b}
$$

let $\varepsilon \rightarrow 0$, we have $|D H(V)|=0$.

Case 4. If $D_{t} H+m(t) H^{2}+B M\left(1+\frac{1}{R^{2}}+\frac{1}{t}\right)=0, \widetilde{H_{a b}} V_{a} V_{b}=0$, then $|D H(V)|=0$. For at $\left(O, R^{2}\right)$ 


$$
\begin{aligned}
& D_{t} H+m(t) H^{2}+B M\left(1+\frac{1}{R^{2}}+\frac{1}{t}\right) \\
= & \Delta H+H\left|H_{a b}\right|^{2}+m(t) H^{2}+B M\left(1+\frac{1}{R^{2}}+\frac{1}{t}\right) \\
\leq & C M\left(1+\frac{1}{R^{2}}+\frac{1}{t}\right)+C M^{3}+B M\left(1+\frac{1}{R^{2}}+\frac{1}{t}\right) \\
\leq & C M+C M^{3} .
\end{aligned}
$$

So for $M \geq 1$,

$$
|D H(V)|^{2} \leq C M^{3} \widetilde{H_{a b}} V_{a} V_{b},
$$

and for $0 \leq M<1$,

$$
|D H(V)|^{2} \leq \widetilde{H_{a b}} V_{a} V_{b} .
$$

REMARK1. If we set $m(t)=0$, then it is obviously satisfies the condition $(\star)$, then we also get the local Harnack under the conditon $0 \leq H_{a b} \leq M g_{a b}$ as a corollary.

\section{Conclusion remarks.}

1. The inequality

$D_{t} H+m(t) H^{2}+2 D H(V)+\left(H_{a b}+m(t) g_{a b}\right) V_{a} V_{b}+B M\left(1+\frac{R^{2}}{\left(R^{2}-4 d^{2}\right)^{2}}+\frac{1}{t}\right) \geq 0$, cannot be got from gradient estimate directly.

Set $V_{a}=-{\widetilde{H_{a b}}}^{-1} D_{b} H$, then

$$
\begin{aligned}
& D_{t} H+m(t) H^{2}+2 D H(V)+\left(H_{a b}+m(t) g_{a b}\right) V_{a} V_{b}+B M\left(1+\frac{R^{2}}{\left(R^{2}-4 d^{2}\right)^{2}}+\frac{1}{t}\right) \\
= & D_{t} H+m(t) H^{2}-{\widetilde{H_{a b}}}^{-1} D_{a} H D_{b} H+B M\left(1+\frac{R^{2}}{\left(R^{2}-4 d^{2}\right)^{2}}+\frac{1}{t}\right),
\end{aligned}
$$

we know $B M\left(1+\frac{R^{2}}{\left(R^{2}-4 d^{2}\right)^{2}}+\frac{1}{t}\right)$ can control $D_{t} H+m(t) H^{2}$ by gradient estimates, but $-\infty \leq-{\widetilde{H_{a b}}}^{-1} \leq-\frac{1}{M+m(t)} g_{a b}$, so $-{\widetilde{H_{a b}}}^{-1} D_{a} H D_{b} H$ can't find the lower bound.

2. It is hard to get $D_{t} H+m(t) H^{2}+2 D H(V)+\left(H_{a b}+m(t) g_{a b}\right) V_{a} V_{b}+B M(1+$ $\left.\frac{R^{2}}{\left(R^{2}-4 d^{2}\right)^{2}}+\frac{1}{t}\right) \geq 0$ directly without using "good extra term". We can have some calculation to show the hard point.

If we set

$$
\begin{gathered}
\widetilde{H_{a b}}=H_{a b}+m(t) g_{a b}, \\
\widetilde{X_{a}}=D_{a} H+\widetilde{H_{a b}} V_{b}, \\
Y_{a b}=D_{a} V_{b}-H H_{a b}, \\
\widetilde{Z}=D_{t} H+2 V_{a} D_{a} H+\widetilde{H_{a b}} V_{a} V_{b} .,
\end{gathered}
$$




$$
\begin{gathered}
W_{a b}=D_{t} H_{a b}+V_{c} D_{c} H_{a b}, \\
W=D_{t} H+V_{c} D_{c} H, \\
\widetilde{U_{a}}=\left(D_{t}-\Delta\right) V_{a}+\widetilde{H_{a b}} D_{b} H .
\end{gathered}
$$

Then

$$
\begin{aligned}
\left(D_{t}-\Delta\right) \widetilde{Z}= & \left|H_{a b}\right|^{2} \widetilde{Z}+2 \widetilde{X_{a}} \widetilde{U_{a}}-2 \widetilde{H_{b c}} Y_{a b} Y_{a c}-4 W_{a b} Y_{a b} \\
& +\left(D_{t} m\right)|V|^{2}+2 m\left(D_{t}-\Delta\right) V_{a} V_{a}-2 m Y_{a b} Y_{a b} \\
& -2 m H^{2}\left|H_{a b}\right|^{2}-4 m H H_{a b} Y_{a b}-m\left|H_{a b}\right|^{2}|V|^{2} \\
& -2 m|D H|^{2}-4 m H_{a b} V_{b} D_{a} H-2 m V_{a}\left(D_{t}-\Delta\right) V_{a} \\
& -2 m^{2} V_{a} D_{a} H+2 m Y_{a b} Y_{a b} . \\
\geq & \left|H_{a b}\right|^{2} \widetilde{Z}+2 \widetilde{X_{a}} \widetilde{U_{a}}-2 \widetilde{H_{b c}} Y_{a b} Y_{a c}-4 W_{a b} Y_{a b}-4 m H H_{a b} Y_{a b} \\
& +\left(D_{t} m-(n+3) m M^{2}\right)|V|^{2}-5 m|D H|^{2}-2 n^{3} m M^{4} .
\end{aligned}
$$

From conditon $(\star), m^{\prime}(t)-(n+3) m M^{2} \geq 0$. When $\widetilde{Z}+\varphi$ attains zero for the first time, we have $\widetilde{X_{a}}=0$, we can let $Y_{a b}=0$. Then

$$
\left(D_{t}-\Delta\right)(\widetilde{Z}+\varphi) \geq\left(D_{t}-\Delta\right) \varphi-5 m|D H|^{2}-\left|H_{a b}\right|^{2} \varphi-2 n^{3} m M^{4} .
$$

We see, all these terms have non-positive lower bound, so we need a "good" term which has big positive lower bound to cancel these terms, this term is in our method.

3 . The additive term $m(t) H^{2}$ is used to make the calculation easier, but it it not the only way, one can add something else.

Acknowledgement. I would like to thank Professor Xi-Ping Zhu, who gave me the problem to do, and he gave me many valuable suggestions and helps during this work. I would like to thank my $\mathrm{PhD}$ advisor Professor KeFeng Liu, who send me to GuangZhou study with Professor Zhu. I also like to thank Professor R.Hamilton, who did the excellent work of local Harnack in Ricci flow, and he gave me some valuable advice. I would like to thank Professor $\mathrm{Mu}$-Tao Wang, who take the notes of R.Hamilton in Columbia, without the notes, I won't do such work.

\section{REFERENCES}

[1] R. Hamilton, The Harnack estimate for the mean curvature flow, J. Diff. Geom., 41 (1995), pp. $215-226$.

[2] R. Hamilton, Curvature and Volume Bounds I, personal communication.

[3] R. Hamilton, Curvature and Volume Bounds II, personal communication.

[4] K. Ecker And G. Huisken, Interior estimates for hypersurfaces moving by mean curvature, Invent. math., 105 (1991), pp. 547-569.

[5] P. Petersen, Riemannian Geometry, Second Edition. 